\title{
Desenvolvimento do predador Podisus nigrispinus alimentado com Spodoptera frugiperda e Tenebrio molitor
}

\author{
Harley Nonato de Oliveira ${ }^{(1)}$, Dirceu Pratissoli(1), Eder Pin Pedruzzi( ${ }^{(1)}$ e Marcelo Curitiba Espindula(1) \\ (1)Universidade Federal do Espírito Santo, Centro de Ciências Agrárias, Dep. de Fitotecnia, Alto Universitário, s/no , CEP 29500-000 Alegre, ES. \\ E-mail: hnoliveira@insecta.ufv.br
}

\begin{abstract}
Resumo - Ninfas de Podisus nigrispinus (Heteroptera: Pentatomidae) têm sido criadas em laboratório com larvas de Tenebrio molitor L. (Coleoptera: Tenebrionidae). No entanto, não existem relatos sobre a predação, no campo ou em laboratório, de P. nigrispinus em Spodoptera frugiperda (Lepidoptera: Noctuidae), uma das principais pragas de inúmeras culturas no Brasil. Este trabalho teve o objetivo de avaliar o desenvolvimento ninfal e características reprodutivas do percevejo predador $P$. nigrispinus em lagartas de $S$. frugiperda e em larvas de T. molitor, em laboratório. A presa S. frugiperda proporcionou ao predador menor longevidade, maior produção e viabilidade de ovos do que as larvas de T. molitor. Esses resultados demonstram que a lagarta S. frugiperda melhora as características reprodutivas de $P$. nigrispinus, de forma que a sua utilização como presa alternativa pode servir para incrementar a produção massal desse inimigo natural.
\end{abstract}

Termos para indexação: controle biológico, percevejo predador, lagarta do cartucho.

\section{Development of the predator Podisus nigrispinus fed on Spodoptera frugiperda and Tenebrio molitor}

\begin{abstract}
Nymphys of Podisus nigrispinus (Heteroptera: Pentatomidae) have been reared on Tenebrio molitor L. (Coleoptera: Tenebrionidae), in laboratory conditions. However, there are no reports on P. nigrispinus predation, in field or laboratory, on Spodoptera frugiperda (Lepidoptera: Noctuidae), one of the most damaging pests in crops in Brazil. This research had the objective to evaluate nymphal development and reproductive characteristics of the predator $P$. nigrispinus when reared on caterpillars of $S$. frugiperda and on larvae of $T$. molitor, in laboratory conditions. S. frugiperda provided a smaller longevity, higher egg production and viability to predator than T. molitor. The nutricional quality of this caterpillar improves the reproductive characteristics of the predator, so that its utilization as factitious host can increase mass production of this natural enemy.
\end{abstract}

Index terms: biological control, stink bugs, beet armyworm.

\section{Introdução}

Várias espécies de percevejos Asopinae são consideradas predadoras de grande utilidade no controle biológico (Molina-Rugama et al., 1998; Oliveira et al., 1999; Vivian et al., 2002). As espécies do gênero Podisus apresentam hábito generalista e são encontradas em diferentes ecossistemas alimentando-se principalmente de larvas de lepidópteros (Zanuncio et al., 1996b; Oliveira et al., 1999), inclusive de lagartas da família Noctuidae (Mohaghegh et al., 2001).

De Clercq \& Degheele (1994) demonstraram o potencial de Podisus maculiventris Say e Podisus sagitta (F.) (Heteroptera: Pentatomidae) no controle de Spodoptera exigua (Hubner) (Lepidoptera: Noctuidae), e Mohaghegh et al. (2001) relataram a capacidade de predação de Podisus nigrispinus (Dallas) (Heteroptera: Pentatomidae) nesse hospedeiro.

Estudos sobre o potencial dos predadores no controle de pragas-chave devem priorizar sua criação massal em laboratório, para que possam ser posteriormente liberados no campo e integrados ao sistema de manejo de pragas. P. nigrispinus tem sido criado e mantido em laboratório com larvas e pupas de Tenebrio molitor (Coleoptera: Tenebrionidae) (Oliveira et al., 2002; Vivan et al., 2002). No entanto, não existem relatos sobre as características biológicas e a predação, no campo ou laboratório, de P. nigrispinus em Spodoptera frugiperda (Smith, 1797) (Lepidoptera: Noctuidae), um inseto polífago relatado como praga de gramíneas como o milho, arroz, capins, cana-deaçúcar e trigo, além de algodão, alface, batata e amendoim, no Brasil (Zucchi et al., 1993; Gallo et al., 2002). 
O objetivo desse trabalho foi estudar o desenvolvimento ninfal e as características reprodutivas de $P$. nigrispinus, em lagartas de $S$. frugiperda e em larvas de T. molitor, em laboratório.

\section{Material e Métodos}

O experimento foi conduzido no Laboratório de Entomologia do Centro de Ciências Agrárias da Universidade Federal do Espírito Santo (CCA-UFES), em Alegre, ES, em sala climatizada com temperatura de $25 \pm 3^{\circ} \mathrm{C}$, umidade relativa de $70 \pm 10 \%$ e fotófase de 14 horas.

A criação de T. molitor foi estabelecida a partir de adultos provenientes do Laboratório de Controle Biológico da Universidade Federal de Viçosa (UFV), Minas Gerais, mantidos em bandejas plásticas de 29×23×11 cm e alimentados com farinha de trigo integral (97\%), levedo de cerveja (3\%) e fatias de chuchu para fornecer a umidade necessária (Zamperline et al., 1992).

$\mathrm{Na}$ criação da presa alternativa $S$. frugiperda, foi utilizada a dieta descrita por Kasten Junior et al. (1978). Ovos da mariposa foram coletados e após a eclosão, três a quatro lagartas foram transferidas para cada tubo de ensaio com $8,5 \mathrm{~cm}$ de altura x 2,3 cm de diâmetro, contendo a dieta. Quando as lagartas transformaramse em pupas, foram transferidas para gaiolas de PVC de $200 \mathrm{~mm}$, com tela de filó nas duas extremidades, para evitar a fuga dos adultos. No interior das gaiolas foram coladas folhas de papel que serviram de local de oviposição. As gaiolas e os tubos foram mantidos em sala climatizada com temperatura de $25 \pm 3^{\circ} \mathrm{C}$, UR $70 \pm 10 \%$ e fotófase de 14 horas.

A criação de $P$. nigrispinus foi iniciada com adultos oriundos da criação da Universidade Federal de Viçosa (UFV), Minas Gerais. Os adultos foram colocados em copos de $500 \mathrm{~mL}$ contendo papel absorvente em seu interior para servir de substrato para oviposição, em número de 8 a 10 por copo. A água era colocada em um tubo, como de anestesia, fixado na borda e coberto em sua extremidade com algodão. $\mathrm{O}$ alimento era constituido de larvas de T. molitor, que foi substituído quando necessário (Zanuncio et al., 2001).

$\mathrm{Na}$ primeira fase do experimento foram realizados dois tratamentos, cada qual com 80 repetições, em que ninfas de segundo estádio foram individualizadas em placas de Petri $(9,0 \times 1,5 \mathrm{~cm})$. Na tampa de cada placa foi fixado um chumaço de algodão $(3 \times 3 \mathrm{~cm})$, previamente comprimido com a mão, e umedecido com água destilada diariamente, para manutenção da umidade e forneci- mento de água. A superfície inferior das placas foi coberta com papel-filtro para absorver o excesso de umidade.

No primeiro tratamento, o predador foi alimentado, em todo o ciclo de vida, com lagartas de quarto estádio de $S$. frugiperda, e no segundo tratamento, com larvas de T. molitor. $\mathrm{O}$ alimento foi conferido diariamente $\mathrm{e}$ trocado quando necessário. Avaliou-se, nesta fase, o peso e o período de duração de cada estádio, além da viabilidade de cada estádio e o total ninfal.

$\mathrm{Na}$ segunda fase do experimento, foram formados vinte casais de adultos recém-emergidos, provenientes da primeira fase, sendo cada casal acondicionado em potes plásticos de $500 \mathrm{~mL}$. Estes potes foram fechados com tampas de plástico nas quais foram colocados tubos do tipo anestesia contendo um chumaço de algodão embebido em água destilada para fornecimento de água aos insetos e manutenção de umidade. O recipiente foi revestido em seu interior com papel ofício, para servir de sítio de oviposição. Os ovos foram coletados diariamente e transferidos para recipientes de plástico $(6 \mathrm{~cm}$ de diâmetro x $2 \mathrm{~cm}$ de altura) contendo um chumaço de algodão umedecido para fornecer umidade aos insetos. A alimentação desta fase foi igual à oferecida nos tratamentos da primeira fase.

Para cada casal individualizado, foram avaliados a longevidade (dias) de fêmeas e machos, o período de préoviposição, o número de posturas, o número de ovos por fêmea e por postura e a viabilidade dos ovos.

O delineamento utilizado foi o inteiramente casualizado; os dados obtidos foram submetidos à análise de variância (ANOVA) e as médias comparadas pelo teste $\mathrm{t}$ a $5 \%$ de probabilidade.

\section{Resultados e Discussão}

A duração do desenvolvimento ninfal não apresentou diferenças significativas entre as presas e variou de 19,81 a 22,51 dias (Tabela 1). Apesar dos diferentes fatores que afetam o desenvolvimento de Asopinae (Moreira et al., 1996; De Clercq et al., 1998; Zanuncio et al., 2001), vários autores verificaram duração da fase ninfal semelhante à observada neste trabalho. Este período foi de aproximadamente 20 dias para P. nigrispinus (Zanuncio et al., 2001), 21 e 22 dias, respectivamente, para fêmeas e machos de Podisus sculptus Distant (Heteroptera: Pentatomidae) (Nascimento et al., 1997), 19 dias para Brontocoris tabidus (Signoret) (Heteroptera: Pentatomidae) (Zanuncio et al., 1996a) e de 19,7 dias para P. nigrispinus (Zanuncio et al., 1996b). 
Esses resultados mostram que espécies de predadores da família Pentatomidae apresentam período de desenvolvimento ninfal de aproximadamente 20 dias, a $25^{\circ} \mathrm{C}$.

A duração do segundo, terceiro e quinto estádio ninfal foi semelhante entre os tratamentos. No entanto, foi verificada diferença significativa entre machos no quarto estádio de desenvolvimento, com menor duração para aqueles que receberam lagartas de $S$. frugiperda. A duração de cada estádio ninfal, quando esse predador foi alimentado com lagartas de $S$. frugiperda, variou de 4,06 a 6,54 dias e de 4,5 a 6,75, com larvas de T. molitor (Tabela 1). Valores semelhantes foram relatados por Zanuncio et al. (1996b), quando criou este mesmo predador com larvas de Musca domestica L., 1758 (Diptera: Muscidae) (3,4 a 6,6 dias) e com larvas de Zophobas confusa Gebien, 1906 (Coleoptera: Tenebrionidae) (3,5 a 7,6 dias). Saini (1994) também encontrou um estádio ninfal semelhante, variando de 3,3 a 6,9 dias quando o predador foi alimentado com larvas de Rachiplusia nu (Guen., 1852) (Lepidoptera: Noctuidae). A semelhança na duração dos estádios indica a adaptação desse predador às diferentes presas e mostra também que a presa utilizada não influencia na duração da fase ninfal.

O tipo de presa não afetou o peso de ninfas de segundo e terceiro estádio do percevejo predador P. nigrispinus. A partir do quarto estádio até o terceiro dia da fase adulta, em ambos tratamentos, houve diferenças entre o peso de machos e fêmeas, com fêmeas apresentando maior peso
(Tabela 1). Zanuncio et al. (1996b) constataram maior peso de fêmeas a partir do quarto estádio ninfal quando criaram $P$. nigrispinus em larvas de Z. confusa. Zanuncio et al. (1998) também observaram fêmeas mais pesadas que machos a partir do quinto estádio com Podisus distinctus (Stal) (Heteroptera: Pentatomidae) alimentados com larvas de T. molitor. Segundo esses autores, esse maior peso é devido ao acúmulo de biomassa necessário para a fase reprodutiva das fêmeas e início de crescimento do sistema reprodutivo das mesmas a partir do quinto estádio.

A viabilidade de $P$. nigrispinus alimentada com S. frugiperda, do segundo ao quinto estádio, variou de $80 \%$ a $95 \%$, e a total foi de $64 \%$. Para os indivíduos alimentados com larvas de T. molitor, essa variação foi de $88 \%$ a 94\% e a total foi de 68\% (Tabela 2). Fernandes et al. (1996) encontraram para P. nigrispinus criado em lagartas de Bombyx mori, viabilidade de $85 \%$ a $96 \%$, do segundo ao quinto estádio, e total de 70\%. Esses mesmos autores observaram para Brontocoris tabidus, viabilidade entre $82 \%$ e $96 \%$ do segundo ao quinto estádio, e total de $57 \%$. Zanuncio et al. (2001) encontraram para P. nigrispinus uma viabilidade total de $73 \%$ quando utilizou $M$. domestica como alimento. Esses resultados são próximos aos encontrados neste trabalho e mostram a adaptação do predador P. nigrispinus à presa S. frugiperda.

A longevidade de adultos de P. nigrispinus somente apresentou variações nas fêmeas, com menor longevidade nas alimentadas com S. frugiperda (Tabela 3). A longevidade dos machos nos dois tratamentos foi seme-

Tabela 1. Desenvolvimento ninfal e peso de ninfas e adultos até o terceiro dia de Podisus nigrispinus alimentados com Spodoptera frugiperda e Tenebrio molitor $^{(1)}$.

\begin{tabular}{|c|c|c|c|c|}
\hline \multirow[t]{2}{*}{ Fase de ínstar e adulta } & \multicolumn{2}{|c|}{ Fêmeas } & \multicolumn{2}{|c|}{ Machos } \\
\hline & Tenebrio molitor & Spodoptera frugiperda & Tenebrio molitor & Spodoptera frugiperda \\
\hline & \multicolumn{4}{|c|}{ Tempo de desenvolvimento (dias) } \\
\hline II & $4,78 \pm 0,43 \mathrm{Aa}$ & $4,31 \pm 0,21 \mathrm{Aa}$ & $4,88 \pm 0,40 \mathrm{Aa}$ & $4,06 \pm 0,17 \mathrm{Aa}$ \\
\hline III & $6,44 \pm 0,75 \mathrm{Aa}$ & $4,69 \pm 0,71 \mathrm{Aa}$ & $5,38 \pm 0,53 \mathrm{Aa}$ & $4,81 \pm 0,47 \mathrm{Aa}$ \\
\hline IV & $4,50 \pm 0,38 \mathrm{Aa}$ & $4,85 \pm 0,47 \mathrm{Aa}$ & $5,50 \pm 0,76 \mathrm{Aa}$ & $4,56 \pm 0,32 \mathrm{Ba}$ \\
\hline $\mathrm{V}$ & $5,89 \pm 0,26 \mathrm{Aa}$ & $6,54 \pm 0,35 \mathrm{Aa}$ & $6,75 \pm 0,45 \mathrm{Aa}$ & $6,38 \pm 0,36 \mathrm{Aa}$ \\
\hline II ínstar - adulto & $21,61 \pm 1,10 \mathrm{Aa}$ & $20,39 \pm 1,26 \mathrm{Aa}$ & $22,51 \pm 1,67 \mathrm{Aa}$ & $19,81 \pm 0,83 \mathrm{Aa}$ \\
\hline \multicolumn{5}{|c|}{ Peso $(\mathrm{mg})$} \\
\hline II & $0,89 \pm 0,04 \mathrm{Aa}$ & $0,92 \pm 0,03 \mathrm{Aa}$ & $0,81 \pm 0,04 \mathrm{Aa}$ & $0,86 \pm 0,06 \mathrm{Aa}$ \\
\hline III & $2,73 \pm 0,20 \mathrm{Aa}$ & $3,18 \pm 0,33 \mathrm{Aa}$ & $2,86 \pm 0,31 \mathrm{Aa}$ & $3,09 \pm 0,40 \mathrm{Aa}$ \\
\hline IV & $9,88 \pm 0,99 \mathrm{Aa}$ & $8,92 \pm 1,30 \mathrm{Aa}$ & $7,25 \pm 0,74 \mathrm{Ab}$ & $7,13 \pm 0,59 \mathrm{Ab}$ \\
\hline V & $27,11 \pm 4,01 \mathrm{Aa}$ & $26,26 \pm 3,13 \mathrm{Aa}$ & $17,86 \pm 2,26 \mathrm{Ab}$ & $19,26 \pm 1,04 \mathrm{Ab}$ \\
\hline $1^{\circ}$ dia adulto & $57,84 \pm 3,49 \mathrm{Aa}$ & $56,18 \pm 3,72 \mathrm{Aa}$ & $36,54 \pm 2,71 \mathrm{Ab}$ & $40,81 \pm 1,76 \mathrm{Ab}$ \\
\hline $2^{2}$ dia adulto & $63,38 \pm 6,84 \mathrm{Aa}$ & $58,10 \pm 5,23 \mathrm{Aa}$ & $38,16 \pm 3,20 \mathrm{Ab}$ & $42,79 \pm 2,34 \mathrm{Ab}$ \\
\hline $3^{o}$ dia adulto & $70,80 \pm 7,54 \mathrm{Aa}$ & $62,34 \pm 5,39 \mathrm{Aa}$ & $37,38 \pm 3,70 \mathrm{Ab}$ & $45,53 \pm 2,14 \mathrm{Ab}$ \\
\hline
\end{tabular}

${ }^{(1)}$ Médias \pm erro-padrão de fêmeas ou de machos tratados com diferentes presas, seguidas de mesma letra maiúscula na linha, não diferem entre si pelo teste t a 5\% de probabilidade; médiasıerro-padrão de machos e fêmeas, tratados com a mesma presa, seguidas de mesma letra minúscula na linha não diferem entre si, pelo teste $\mathrm{t}$ a $5 \%$ de probabilidade. 
lhante, assim como os resultados obtidos por Nascimento et al. (1997) para P. nigrispinus criados em larvas de T. molitor e lagartas de Bombyx mori. Porém, a menor longevidade de fêmeas criadas com S. frugiperda concorda com os resultados obtidos por Zanuncio et al. (2001) na criação de $P$. nigrispinus com pupas de T. molitor (35,3 dias) e com larvas de $M$. domestica (29,7 dias). Essa menor longevidade com lagartas de $S$. frugiperda talvez possa ser explicada pelo fato de que essas fêmeas, quando alimentadas nesta presa apresentaram um acentuado incremento no número de ovos e no número de ovos por postura, diferindo estatisticamente daquelas alimentadas com T. molitor. Com esse aumento na oviposição, ocorreu um acentuado gasto de energia, resultando em redução da sua longevidade. Segundo Sibly \& Calow (1986), isto é possível, uma vez que há troca na alocação de fontes entre diferentes processos fisiológicos, ou seja, o incremento de algum processo (por exemplo, produção de ovos) é contraposto a uma diminuição da alocação de recursos energéticos de outro (longevidade).

As fêmeas dos diferentes tratamentos não diferiram entre si no período de pré-oviposição e número de posturas. No entanto, o número de ovos por fêmea, número de

Tabela 2. Viabilidade (\%) ninfal de Podisus nigrispinus (Heteroptera: Pentatomidae) alimentados com lagartas de Spodoptera frugiperda (Lepidoptera: Noctuidae) e larvas de Tenebrio molitor (Coleoptera: Tenebrionidae).

\begin{tabular}{ccc}
\hline \multirow{2}{*}{ Ínstar } & \multicolumn{2}{c}{ Podisus nigrispinus } \\
\cline { 2 - 3 } & Spodoptera frugiperda & Tenebrio molitor \\
\hline II & $90 \%$ & $88 \%$ \\
III & $80 \%$ & $90 \%$ \\
IV & $94 \%$ & $90 \%$ \\
V & $95 \%$ & $94 \%$ \\
\hline Total & $64 \%$ & $68 \%$ \\
\hline
\end{tabular}

Tabela 3. Parâmetros avaliados em fêmeas de Podisus nigrispinus alimentadas com Spodoptera frugiperda e Tenebrio molitor ${ }^{(1)}$.

\begin{tabular}{lcc}
\hline Parâmetros & $\begin{array}{c}\text { Spodoptera } \\
\text { frugiperda }\end{array}$ & $\begin{array}{c}\text { Tenebrio } \\
\text { molitor }\end{array}$ \\
\hline Período de pré-oviposição (dias) & $7,22 \pm 0,72 \mathrm{~A}$ & $7,88 \pm 0,88 \mathrm{~A}$ \\
Número de posturas & $14,89 \pm 3,77 \mathrm{~A}$ & $17,00 \pm 2,59 \mathrm{~A}$ \\
Número de ovos por fêmea & $447,62 \pm 27,65 \mathrm{~A}$ & $325,00 \pm 31,38 \mathrm{~B}$ \\
Número de ovos por postura & $30,06 \pm 0,97 \mathrm{~A}$ & $19,06 \pm 0,99 \mathrm{~B}$ \\
Viabilidade dos ovos (\%) & $85,19 \pm 1,50 \mathrm{~A}$ & $79,25 \pm 1,85 \mathrm{~B}$ \\
Longevidade (fêmeas) & $39,6 \pm 7,38 \mathrm{~B}$ & $67,2 \pm 7,49 \mathrm{~A}$ \\
Longevidade (machos) & $56,6 \pm 5,34 \mathrm{~A}$ & $49,5 \pm 7,30 \mathrm{~A}$ \\
\hline
\end{tabular}

${ }^{(1)}$ Médias seguidas de mesma letra na linha não diferem entre si, pelo teste $\mathrm{t}$ a $5 \%$ de probabilidade. ovos por postura e viabilidade dos ovos foram diferentes entre os tratamentos, e os maiores valores foram observados em fêmeas de $P$. nigrispinus alimentadas com lagartas de $S$. frugiperda (Tabela 3 e Figura 1). Essas fêmeas produziram uma média de 122 ovos a mais do que quando alimentadas com T. molitor (Tabela 3). A média do número de ovos por fêmea, em S. frugiperda (447 ovos) foi maior do que os valores encontrados por Oliveira et al. (2002) na criação de P. nigrispinus com pupas de T. molitor, com disponibilidade de alimentação em plantas de tomate, e semelhante aos valores relatados por Zanuncio et al. (2001), quando esse predador alimentou-se da combinação de pupas de T. molitor e larvas de $M$. domestica. Esses autores relataram que nessa combinação de presas, as exigências nutricionais de $P$. nigrispinus são suplementadas, proporcionando um maior número de ovos por fêmea. Dessa forma, lagartas de $S$. frugiperda, possivelmente por possuirem melhor qualidade nutricional, podem proporcionar às fêmeas de $P$. nigrispinus uma produção de ovos semelhante à daquelas alimentadas com uma combinação de presas. Além dessa melhor qualidade nutricional, segundo De Clercq \& Degheele (1994), Podisus maculiventris (Say) e Podisus sagitta (F.) (Heteroptera: Pentatomidae) apresentam alto potencial de predação de lagartas de S. exigua (Hubner) (Lepidoptera: Noctuidae), em condições de laboratório, mostrando que podem ser utilizados como agente de controle biológico dessa praga.

Além disso, S. frugiperda apresenta a vantagem de ser facilmente criada em laboratório e poder ser utilizada quando o objetivo for incrementar a criação do percevejo predador em menor período de tempo.

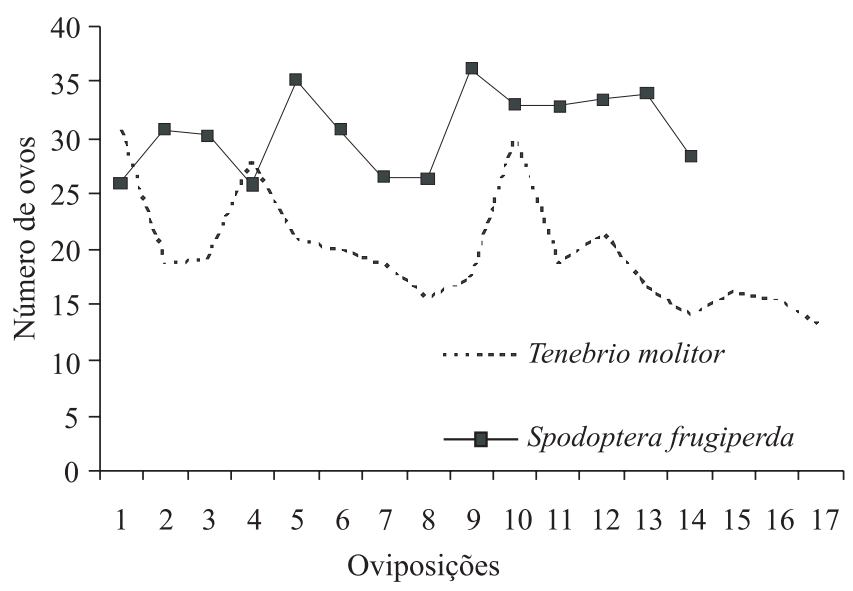

Figura 1. Número médio de ovos de Podisus nigrispinus (Heteroptera: Pentatomidae), por oviposição, alimentado com Tenebrio molitor (Coleoptera: Tenebrionidae) e Spodoptera frugiperda (Lepidoptera: Noctuidae). 


\section{Conclusão}

A presa $S$. frugiperda proporcionou melhores características reprodutivas ao predador $P$. nigrispinus, e pode ser utilizada quando houver necessidade de incrementar a criação desse predador em laboratório, em um reduzido intervalo de tempo.

\section{Agradecimentos}

Ao Conselho Nacional de Desenvolvimento Científico e Tecnológico, pelo auxílio financeiro e pela bolsa concedida ao autor Harley Nonato de Oliveira.

\section{Referências}

DE CLERCQ, P.; DEGHEELE, D. Laboratory measurement of predation by Podisus maculiventris and P. sagitta (Hemiptera: Pentatomidae) on beet armyworm (Lepidoptera: Noctuidae). Journal of Economic Entomology, v.87, p.76-83, 1994.

DE CLERCQ, P.; VANDEWALLE, M.; TIRRY, L. Impact of inbreeding on performance of the predator Podisus maculiventris (Heteroptera: Pentanomidae). Biocontrol, v.43, p.299-310, 1998.

FERNANDES, L.G.; CARVALHO, C.F.; BUENO, V.H.P.; DINIZ, L.C. Aspectos biológicos de Brontocoris tabidus Signoret, 1852 e Podisus nigrispinus Dallas (Hemiptera: Pentatomidae). Cerne, v.2, p.1-15, 1996.

GALLO, D.; NAKANO, O.; SILVEIRA NETO, S.; CARVALHO, R.P.L.; BATISTA, G.C. de; BERTI FILHO, E.; PARRA, J.R.P.; ZUCCHI, R.A.; ALVES, S.B.; VENDRAMIM, J.D.; MARCHINI, L.C.; LOPES, J.R.S.; OMOTO, C. Entomologia agrícola. São Paulo: Fealq, 2002. 920p.

KASTEN JÚNIOR, P.; PRECETTI, A.A.C.M.; PARRA, J.R.P. Dados biológicos comparativos de Spodoptera frugiperda (J.E. Smith, 1797) em duas dietas artificiais e substrato natural. Revista de Agricultura, v.53, p.68-78, 1978.

MOHAGHEGH, J.; DE CLERCQ, P.; TIRRY, L. Functional response of the predators Podisus maculiventris (Say) and Podisus nigrispinus (Dallas) (Heteroptera: Pentatomidae) to the beet armyworm, Spodoptera exigua (Hubner) (Lepidoptera: Noctuidae): effect of temperature. Journal of Applied Entomology, v.125, p.131134, 2001.

MOLINA-RUGAMA, A.J.; ZANUNCIO, J.C.; PRATISSOLI, D.; CRUZ, I. Efeito do intervalo de alimentação na reprodução e na longevidade do predador Podisus nigrispinus (Dallas) (Heteroptera: Pentatomidae). Anais da Sociedade Entomológica do Brasil, v.27, p.77-84, 1998.

MOREIRA, L.A.; ZANUNCIO, J.C.; PICANÇO, M.C.; GUEDES, R.N.C. Effect of Eucalyptus feeding in the development, survival and reproduction of Tynacantha marginata (Heteroptera: Pentatomidae). Revista de Biologia Tropical, v.44, p.253-257, 1996.

NASCIMENTO, E.C.; ZANUNCIO, J.C.; PICANÇO, M.C.;
ZANUNCIO, T.V. Desenvolvimento de Podisus sculptus Distant, 1889 (Heteroptera: Pentatomidae) em Bombyx mori (Lepidoptera: Bombycidae) e Tenebrio molitor (Coleoptera: Tenebrionidae). Revista Brasileira de Biologia, v.57, p.195-201, 1997.

OLIVEIRA, H.N.; ZANUNCIO, J.C.; SOSSAI, M.F.; PRATISSOLI, D. Body weight increment of Podisus nigrispinus (Stal) (Heteroptera: Pentatomidae), fed on Tenebrio molitor L. (Coleoptera: Tenebrionidae) or Musca domestica L. (Diptera: Muscidae). Brenesia, v.51, p.77-83, 1999.

OLIVEIRA, J.E.M.; TORRES, J.B.; CARRANO-MOREIRA, A.F.; BARROS, R. Efeito das plantas do algodoeiro e do tomateiro, como complemento alimentar, no desenvolvimento e na reprodução do predador Podisus nigrispinus (Dallas) (Heteroptera: Pentatomidae). Neotropical Entomology, v.31, p.101-108, 2002.

SAINI, E.D. Aspectos morfologicos y biologicos de Podisus connexivus Bergroth (Heteroptera: Pentatomidae). Revista de la Sociedad Entomológica Argentina, v.53, p.35-42, 1994.

SIBLY, R.M.; CALOW, P. Physiological ecology of animals: an evolutionary approach. Oxford: Blackwell Scientific Publications. 1986. 179p.

VIVIAN, L.M.; TORRES, J.B.; BARROS, R.; VEIGA, A.F.S.L. Tasa de crecimiento poblacional del chinche depredador Podisus nigrispinus (Heteroptera: Pentatomidae) y de la presa Tuta absoluta (Lepidoptera: Gelechiidae) en invernadero. Revista de Biologia Tropical, v.50, p.145-153, 2002.

ZAMPERLINE, B.; ZANUNCIO, J.C.; LEITE, J.E.M.; BRAGANÇA, M.A.L. Influência da alimentação de Tenebrio molitor L. 1758 (Coleoptera:Tenebrionidae) no desenvolvimento ninfal de Podisus connexivus Bergroth, 1891 (Hemiptera: Pentatomidae). Revista Árvore, v.16, p.224-230, 1992.

ZANUNCIO, J.C.; MOLINA-RUGAMA, A.J.; SERRÃO, J.E.; PRATISSOLI, D. Nymphal development and reproduction of Podisus nigrispinus (Heteroptera: Pentatomidae) fed with combinations of Tenebrio molitor (Coleoptera: Tenebrionidae) pupae and Musca domestica (Diptera: Muscidae) larvae. Biocontrol Science and Technology, v.11, p.331-337, 2001.

ZANUNCIO, J.C.; SAAVEDRA, J.L.D.; OLIVEIRA, H.N.; DEGHEELE, D.; DE CLERCQ, P. Development of the predatory stinkbug Brontocoris tabidus (Signoret) (Heteroptera: Pentatomidae) on different proportions of an artificial diet and pupae of Tenebrio molitor L. (Coleoptera: Tenebrionidae). Biocontrol Science and Technology, v.6, p.619-625, 1996a.

ZANUNCIO, T.V.; TORRES, J.B.; ZANUNCIO, J.C.; SANTOS, G.P. Ciclo de vida e reprodução de Podisus distinctus (Stal) (Heteroptera: Pentatomidae) alimentado com dois tipos de presas. Revista Brasileira de Entomologia, v.41, p.335-337, 1998.

ZANUNCIO, T.V.; ZANUNCIO, J.C.; SAAVEDRA, J.L.D.; LOPES, E.D. Desenvolvimento de Podisus nigrispinus (Dallas) (Heteroptera: Pentatomidae) com Zophobas confusa Gebien (Coleoptera: Tenebrionidae) comparado a duas outras presas alternativas. Revista Brasileira de Zoologia, v.13, p.159-164, 1996b.

ZUCCHI, R.A.; SILVEIRA NETO, S.; NAKANO, O. Guia de identificação de pragas agrícolas. São Paulo: Fealq, 1993. 139p.

Recebido em 12 de dezembro de 2003 e aprovado em 15 de julho de 2004

Pesq. agropec. bras., Brasília, v.39, n.10, p.947-951, out. 2004 